\title{
The Research of Removing Elemental Mercury in Flue Gas by Peroxidase-like catalyst
}

\author{
Bohua Sun
}

\author{
School of North China Electric Power University(Baoding), Baoding 071003, China
}

Keywords: peroxidase-like catalyst,elemental mercury, mercury removal efficiency, chemical analysis

\begin{abstract}
In this paper,peroxidase-like catalyst was prepared by organic synthesis. The removal of elemental mercury in flue gas and the effect of single factor experiments were carried out in a simulated flue gas system. The result shows that mercury removal efficiency could reach $70.6 \%$ when $30 \%$ hydrogen peroxide $\left(\mathrm{H}_{2} \mathrm{O}_{2}\right)$ addition amount is $9.8 \mathrm{mmol}$, the amount of the peroxidase-like catalyst is $0.02 \mathrm{~g}$, and the initial $\mathrm{pH}$ value of the solution is 8.81 and the reaction temperature is $40.63{ }^{\circ} \mathrm{C}$. Besides, catalyst oxidation products of mercury removal experiment were analyzed by atomic fluorescence spectrophotometer. In addition, Isopropyl alcohol was used as the capture agent of hydroxyl radical produced by hydrogen peroxide. Strong oxidizing substances produced by peroxidase-like catalyst and hydrogen peroxide is high valence iron and hydroxyl radicals, in which high valence iron plays a major role, hydroxyl radical and hydrogen peroxide play a secondary role. Generally speaking, the three compound act together to oxidize the elemental mercury $\left(\mathrm{Hg}^{0}\right)$ into two valent mercury $\left(\mathrm{Hg}^{2+}\right)$.
\end{abstract}

\section{Introduction}

In China, thermal power plant occupies an important position in the national flue gas emissions. More than $82 \%$ of the electricity is produced by burning fossil fuels. However, the trace elements of mercury in coal, which has a serious pollution to the environment is difficult to completely remove. In addition, its potential and conceal influence to the entire ecosystem can not be ignored[1].

There are many kinds of mercury in boiler flue gas discharged from thermal power plant, the main components are gaseous elemental mercury $\left(\mathrm{Hg}^{0}\right)$, gaseous two $\left(\mathrm{Hg}^{2+}\right)$ and solid particulate mercury $\left(\mathrm{Hg}^{\mathrm{P}}\right)$. Elemental mercury is the most important source of coal combustion emissions. However, most of the elemental mercury is stable due to its nature character, which is inert and not easy to remove. At present,changing the elemental mercury into two valence mercury is the main research object of mercury[2].

There are a lot of researches on mercury removal from coal-fired flue gas, but most of them are in the laboratory stage. The peroxidase like catalyst is a chemical substance which not only has the high efficiency of biological enzyme, but also overcomes the specificity of biological enzyme action. Therefore, it is a good choice to study the use of peroxidase to catalyze mercury. In order to solve the problem of mercury pollution in the flue gas of coal-fired power plant, this paper puts forward the method of liquid phase catalytic oxidation to remove elemental mercury.

\section{Preparation and Characterization of Enzyme Like Catalysts}

\subsection{Preparation of Enzyme Like Catalysts}

By using organic method, the transition metal ions in different transition states of iron, cobalt and nickel are synthesized as the central atom, and an enzyme like catalyst is formed around the 4 nitrogen atoms. The preparation process can be divided into three steps. The specific reaction equation is as follows.

- The first step: 


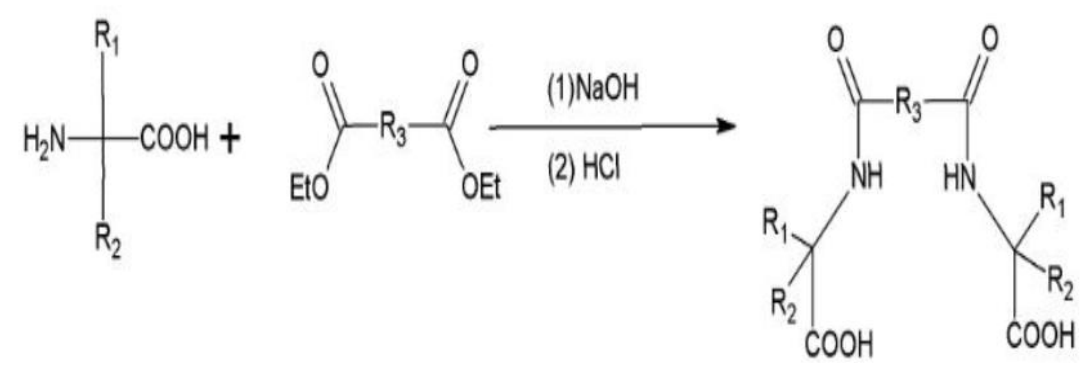

- The second step:<smiles>[R]C([R])(NC(=O)[B]C(=O)NC([R])([R])C(=O)O)C(=O)O</smiles><smiles></smiles>

- The third step:

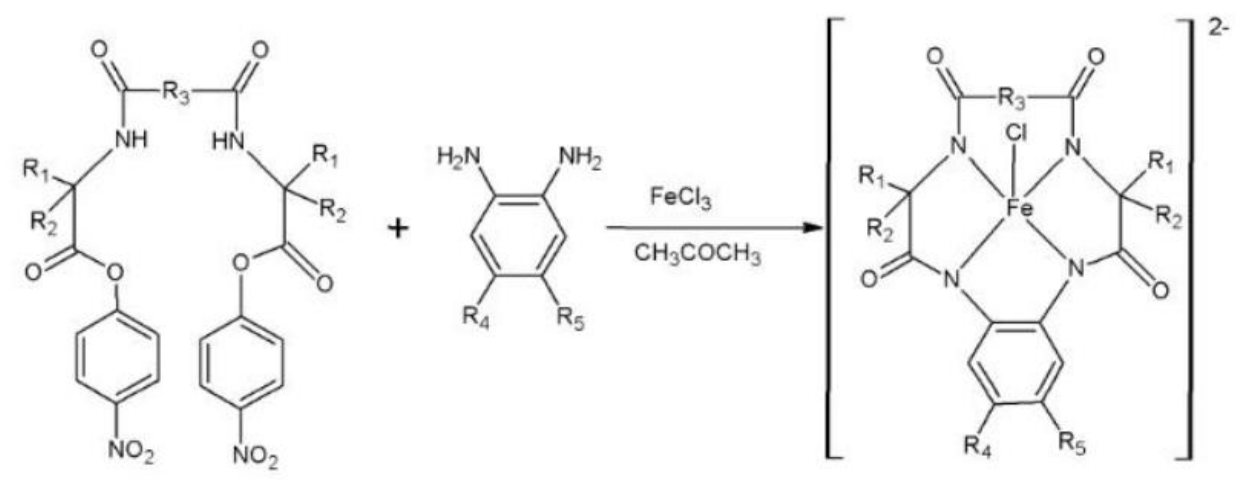

\subsection{Characterization of Enzyme Like Catalysts}

In order to analyze the physical and chemical properties of peroxidase, the catalyst was characterized by UV Vis spectrophotometer. Take $0.02 \mathrm{~g}$ class peroxidase and put it into the $50 \mathrm{ml}$ beaker. Add deionized water to dissolve it. After standing for 10 minutes, with a syringe filter absorbs the supernatant dripping into the cuvette, and then use the T6 series UV to measure it. UV absorption spectra of synthetic materials is shown in Fig 1.

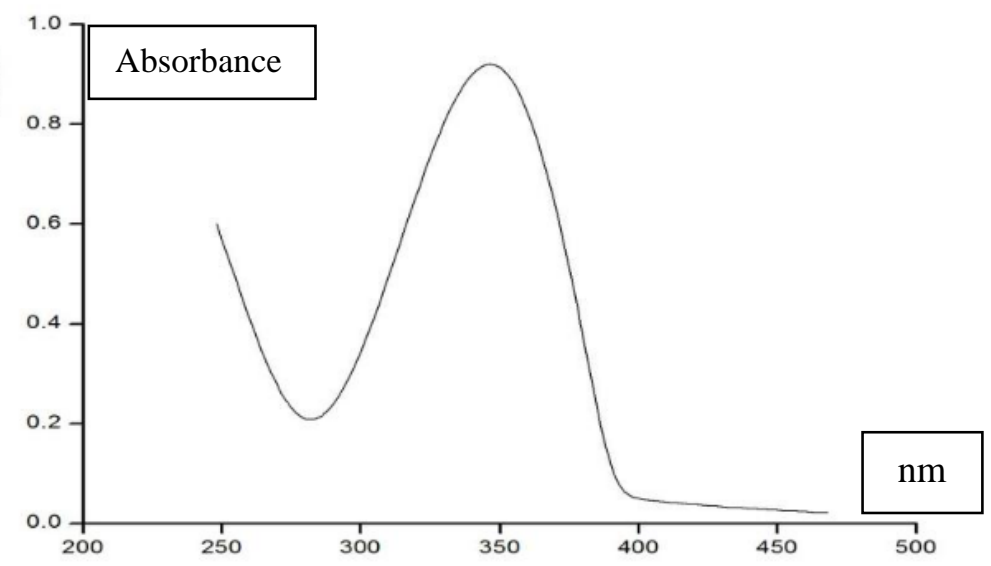

Fig. 1: UV absorption spectra of synthetic materials 
Fig. 1 indicates that the catalyst's absorption peak is near 350nm. Meanwhile, UV Fe-TAML and related information in the visible light absorption peak is near $368 \mathrm{~nm}$ coincide, which proves that the catalyst is a kind of peroxidase-like catalyst.

\section{The Experiment of Removing Elemental Mercury In Flue Gas By Peroxidase-like}

The removal efficiency of the experiment used a homemade drum to study peroxidase-like liquid phase catalytic oxidation of elemental mercury in flue gas bubble reactor. The experimental system process is shown in Fig. 2 which is mainly composed of flue gas generated system, mercury vapor generating device, gas analysis system, the tail gas absorption system components.

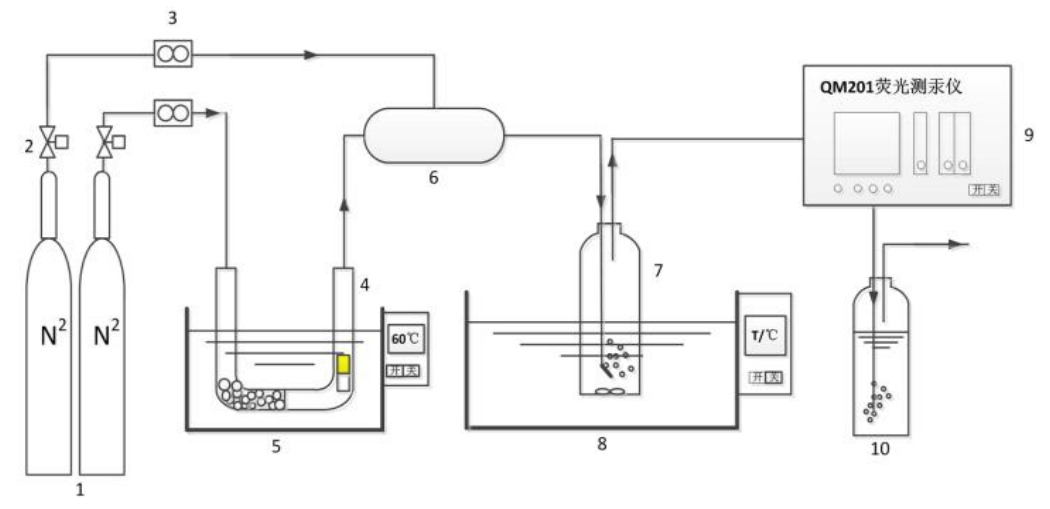

Fig. 2: The experimental system process

Test principle: the carrier gas $\left(\mathrm{N}_{2}\right)$ will be loaded into the pool of mercury vapor fluorescence, mercury vapor in the low pressure mercury lamp 253.7 sharp line light excitation, The fluorescent light converging to the photocathode of photomultiplier tube and is converted into an electrical signal. Furthermore, the electrical signal is magnified by the measurement results of the fluorescence mercury measurement instrument[3]. The fluorescence intensity is proportional to the concentration of atoms under certain conditions.

Based on the single factor analysis, we obtained the optimum amount of hydrogen peroxide, the amount of catalyst, the $\mathrm{pH}$ value and the temperature of the catalyst. That is $30 \%$ hydrogen peroxide

$\left(\mathrm{H}_{2} \mathrm{O}_{2}\right)$ addition amount is $9.8 \mathrm{mmol}$, the amount of the enzyme catalyst is $0.02 \mathrm{~g}$, and the initial $\mathrm{pH}$ value of the solution is 8.81 and the reaction temperature is $40.63{ }^{\circ} \mathrm{C}$.

\section{Study and Analysis on the reaction mechanism of mercury removal}

At present, there are three kinds of high efficient catalytic oxidation of elemental mercury. First, Fenton's reagent is an oxidation system composed of hydrogen peroxide and catalyst Fe. Under the action of catalyst Fe, hydrogen peroxide can produce hydroxyl radical with strong oxidation ability, which accelerates the oxidation of elemental mercury [4]; Second, the type of fenton reagent is composed of $\mathrm{Fe} 3$ + catalyst and hydrogen peroxide oxidation system. Under the effect of catalyst Fe3+. Hydrogen peroxide can produce highly oxidizing hydroxyl radicals and peroxy radicals, thus speeding up the oxidation of elemental mercury; Third, the type of peroxidase-like catalysts are macrocyclic metal complexes. On the one hand, hydrogen peroxide effect makes hydrogen peroxide into hydroxyl radicals. On the other hand ,it also generates oxidizing strong high iron [5]. Besides, isopropyl alcohol can be used as the hydroxyl free radical trapping agent [6].

According to the above analysis, we design three groups experiments. The first experiment group added $1 \mathrm{ml} 30 \%$ hydrogen peroxide; The second one adds $1 \mathrm{ml} \mathrm{30 \%} \mathrm{hydrogen} \mathrm{peroxide} \mathrm{and} \mathrm{a} \mathrm{certain}$ amount of acetone isopropanol; The third group only $1 \mathrm{ml} \mathrm{30 \%} \mathrm{addition} \mathrm{of} \mathrm{hydrogen} \mathrm{peroxide} \mathrm{without}$ catalyst and should be quickly done to prevent the rapid oxidation of hydrogen peroxide. Furthermore, The bubbling reactor is connected to the gas path in order to remove mercury. The reaction time is $30 \mathrm{~min}$. Record the reading of the fluorescence mercury meter[7]. The mercury removal efficiency of the three groups were $70.51 \%$, 58.5\%, and $28.97 \%$, respectively. 
It can be inferred from the experimental data that there are not only hydroxyl radical, but also high valence iron in the strong oxidizing substance produced by the enzyme like catalyst and hydrogen peroxide. Hydrogen peroxide can also oxide mercuryl, but the efficiency is not very high, after adding the peroxidase-like catalyst, removal mercury efficiency is significantly improved.

\section{Conclusions}

To sum up, the best experimental conditions for the catalytic oxidation of elemental mercury in flue gas by enzyme like catalyst were obtained. Study on the reaction mechanism of isopropanol as hydroxyl radical scavenger. Under the same conditions, The efficiency of mercury removal by adding acetone was only about $12 \%$ lower than that without adding acetone. Meanwhile, the mercury removal efficiency was higher than that of only adding hydrogen peroxide. It suggests that the main role of high valence iron in the strong oxidizing substances produced by the enzyme like catalyst and hydrogen peroxide. In the same time, high valence iron plays a major role in the oxidation of hydroxyl radical and hydrogen peroxide. Hydroxyl radicals and hydrogen peroxide plays a minor role. The three compound act together to oxidize the elemental mercury into two valent mercury .

\section{References}

[1] Xu Yongyi, Zha Zhi Ming, Huang Qishun. A brief introduction of flue gas mercury removal technology [J]. industrial safety and environmental protection, 2007, 33 (10)

[2] Ren Jianli, Zhou Jingsong, et al. Experimental study on mercury emission from coal combustion process [J]. Journal of: Engineering Science, Zhejiang University (in Chinese)edition

[3] Dong Liyan. Experimental study on flue gas mercury removal by gas phase oxidation [D].

[4] Zhang Xing, Liang Damei, Liu Jing,, et al. Analysis of the removal efficiency of elemental mercury oxidation by reagent,, heat, [J]Power generation, 2005, 44, ():51-55.

[5]Brown T D, Smith D N, Hargis J R A, et a1. measurement its What we know control:, have learned, and need further investi. Journal of the Air \& Waste to MercuryManagement Association, 1999 (6):628-640.Experimental study on degradation of organic pollutants in aqueous solution by

[6] Cui Yingjie. [D]. Qingdao, environmental engineering, 2008.5., Feton

[7]Laudal D.L., Brown T.D., Nott B.R.Effects flue. constituents mercury speciation[J].Fuel Technology Processing, 2000, 66:57-165., on, of, gas 